\title{
THE USE OF OPTICAL METHODS FOR LEAK TESTING DAMPERS
}

\author{
TOMASIAK, J.
}

Abstract: The present paper presents the possible use of a portable structural light $3 D$ scanner for performing measurements in manufacturing environments. A measurement analysis of the geometry of a damper was performed in order to check the leak-tightness of the damper. The data obtained, having the form of a point cloud, was processed and a triangular network was built and compared with a virtual $3 D$ model. The results that were obtained constituted a basis for identification of measurement areas. Then the concept was proposed to create a model surface in order to reduce the measurement uncertainty.

Key words: 3D scanning, point cloud, optical measurement, surface texture
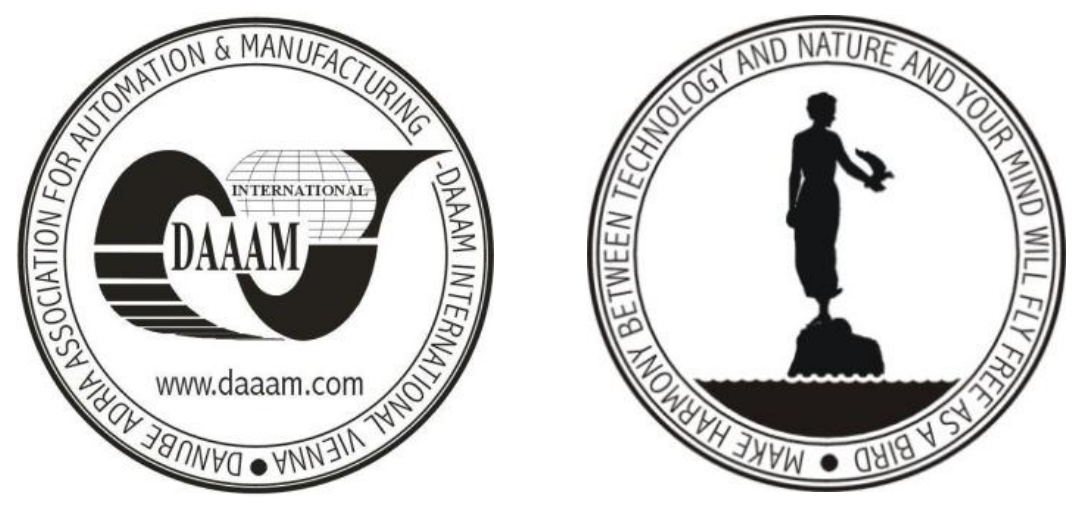

Authors' data: M.Sc. Eng. Tomasiak, J[acek]*; The school of higher vocational education in nysa, Armii Krajowej 7, 48-300, Nysa, Poland, jacek.tomasiak@pwsz.nysa.pl

This Publication has to be referred as: Tomasiak, J[acek] (2013) The Use of Optical Methods for Leak Testing Dampers, Chapter 47 in DAAAM International Scientific Book 2013, pp. 787-794, B. Katalinic \& Z. Tekic (Eds.), Published by DAAAM International, ISBN 978-3-901509-94-0, ISSN 1726-9687, Vienna, Austria DOI: $10.2507 /$ daaam.scibook.2013.47 


\section{Introduction}

Contemporary optical 3D scanning techniques are one of the fastest growing fields of metrology. Nowadays the industry requires accurate measurements of products performed in the shortest possible time and that can be use of optical measurement methods enables this. Optical measurements result in the collection of data in the form of a point cloud. The results obtained, i.e. the 3D points, are then matched and connected by using appropriate algorithms, then a triangular network is generated on their basis. The quality of the scanning process and the process of matching of individual elements depends on the repeatability of the rotation of the item or the order in which, for example, the scanner's head moves. When high repeatability can be achieved, it is possible to introduce algorithms that build a model based on averaged data from many series. Such high precision is guaranteed only by expensive commercial solutions. However, averaging algorithms are an integral part of the software of such a scanner and cannot be customized by the user (Kesik, 2011). The use of optical measurement methods enables to achieve comprehensive information about the measured object in a short time. This is particularly important in the case of large objects or free surfaces.

The article presents the use of an optical measurement method for the purpose of analysis of leak-tightness of a damper and the concepts of a model surface intended to reduce the measurement uncertainty.

\section{Description of the Test}

The objective of the test was to check the shape of the surface of the seat of a damper used in the chemical industry (Fig. 1). The accuracy with which the tested internal surface was made has a significant impact on the leak-tightness of the damper. Due to the dimensions of the measured object, a 3D scanning technique was used to evaluate the shape of the surface.

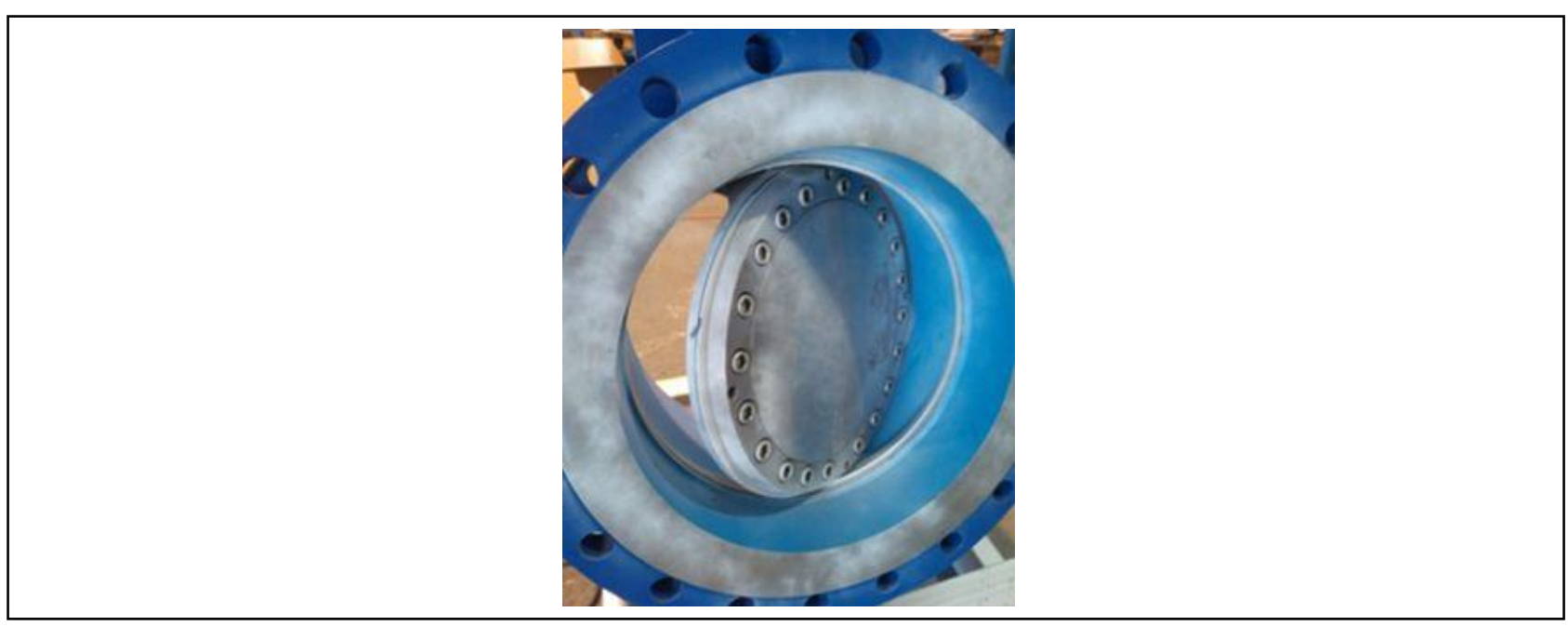

Fig. 1. Tested object 
The geometry of the object was measured using the ATOS Triple Scan optical scanner made by GOM, which is shown in Fig. 2. The scanner uses the blue light technology. The narrow-band blue light enables precise measurements regardless of the external lighting conditions.

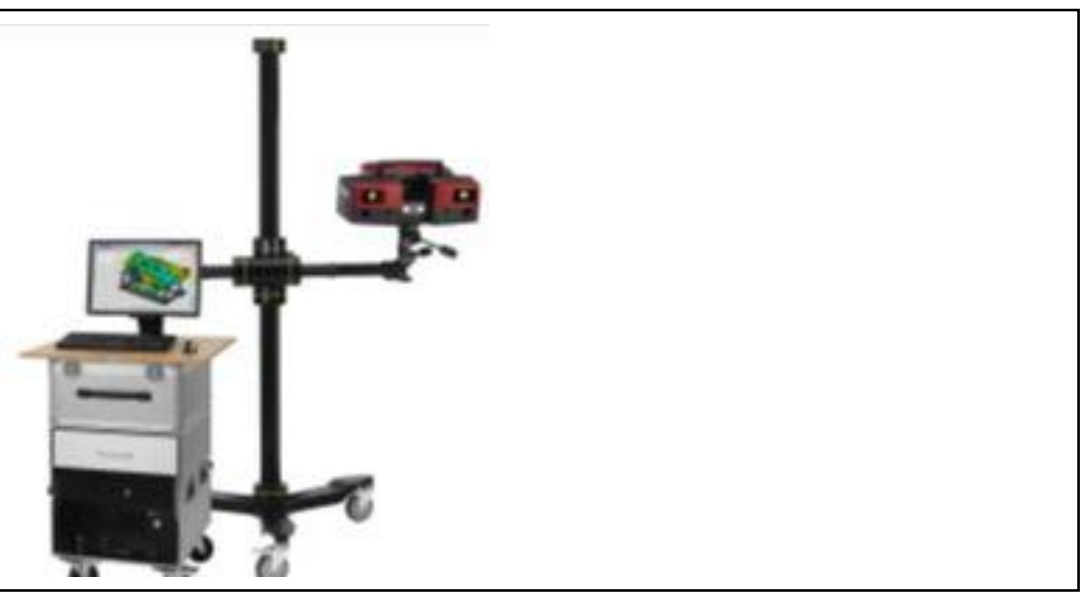

Fig. 2. ATOS Triple Scan made by GOM

The scanner uses two up to $8 \mathrm{MB}$ high-resolution cameras and a projector. As a result, during a single measurement, the scanner can record a larger measurement area with simultaneous reduction of the number of scanner positions (www.gom.com). The use of a system of two cameras increases the depth of measurement of holes which is appropriate for smaller triangulation angles (Fig. 3).

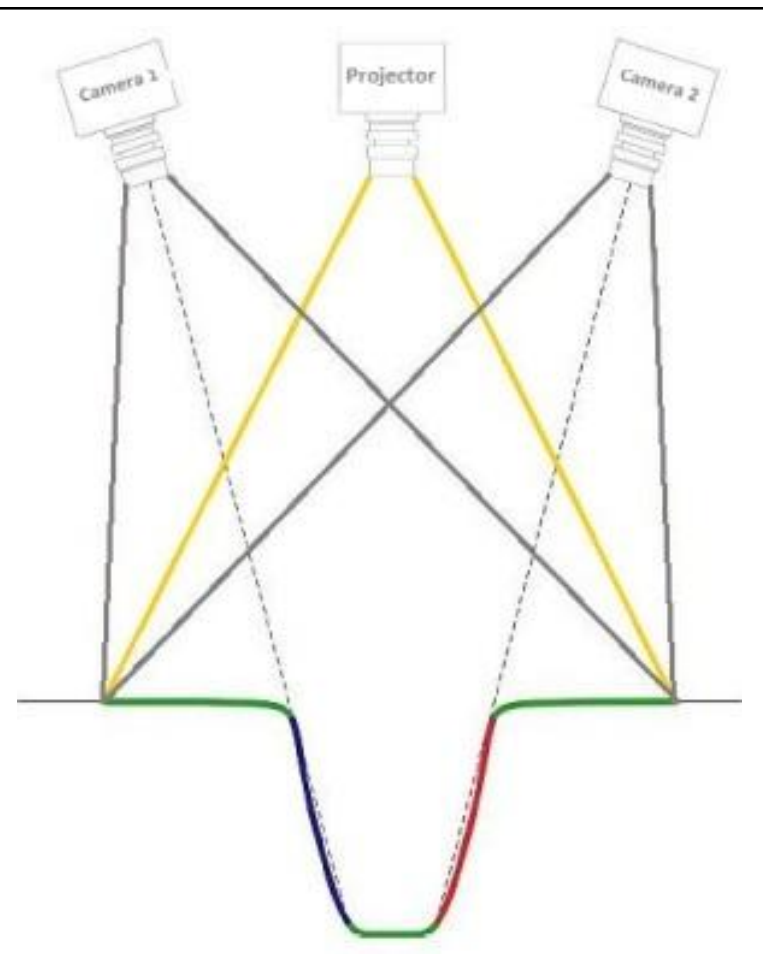

Fig. 3. ATOS measurement system: green area - measurement data from both cameras; red area - measurement data from the projector and the right camera; blue area - measurement data from the projector and the left camera 
The flexibility of the scanner allows to measure a very large spectrum of details using the same head and the additional use of the TRITOP photogrammetric system enables the ATOS to scan over 30-meter details with high local accuracy maintained.

Table 1 shows the parameters of the scanner used for the test.

\begin{tabular}{|l|l|}
\hline Parameter & Value \\
\hline Camera Pixels & $2 \times 8000000$ \\
\hline Measuring Area & $38 \times 29-2000 \times 1500 \mathrm{~mm}^{2}$ \\
\hline Point Spacing & $0.01-0.61 \mathrm{~mm}$ \\
\hline Working Distance & $490-2000 \mathrm{~mm}$ \\
\hline Sensor Dimensions & $340 \mathrm{~mm} \times 130 \mathrm{~mm} \times 230 \mathrm{~mm}$ \\
\hline
\end{tabular}

Tab.1. Characteristics of the ATOS scanner

\subsection{Test procedure}

The objective of the test was to perform measurements of the seat of a damper and to find machining defects that have a significant impact on the leak-tightness of the damper.

The measurements were performed in the following stages:

- Calibration of the measurement head.

- Scanning of the real object.

- Processing of the point cloud.

- Importation of the CAD 3D model.

- Matching the scanned model with the CAD 3D model.

- Comparison of the superimposed models and analysis.

The scanning process was preceded by calibration of the instrument and preliminary preparation of the surface of the tested product, which consisted of cleaning and attaching the check points (markers). Due to the fact that during the scanning the surface of the seat of the damper reflected excessive amounts of light, it was covered with sprayed chalk.

The 3D scanning process consisted of mapping of the shape of the real object (the interior of the damper) and recording it in a digital form. The projector of the scanner transferred a set of lines of known density onto the tested object. The straight lines were then deformed to the extent that was proportional to the deformation of the object's surface and this image was recorded by the cameras. Using the input data (the structure of the light, the image recorded by the camera, the camera's calibration parameters, the angle between the projection direction and the readout direction) and the relevant mathematical formulas, the coordinates of each camera pixel were calculated. The result of a single measurement is a cloud of points whose number of which depends directly on the resolution of the cameras used (Fig. $4)$. 


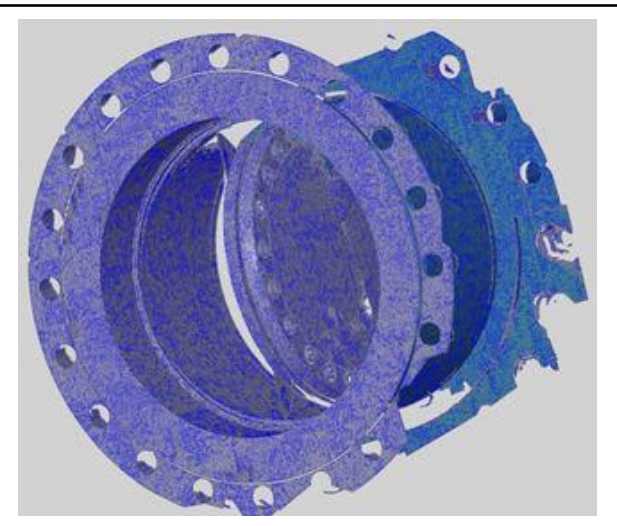

Fig. 4. Point cloud

The point cloud was then processed using the GOM Inspect V7.5 software. A standard procedure followed when working on a point cloud is to create a triangular network. It is intended to reduce the size of the file by connecting the points into triangles and eliminating those points that do not provide the required additional information. In practice, the number of points located in a smooth surface are significantly reduced while the density of the points on the edges remains practically unchanged (Rak \& Wozniak, 2013). The triangular network is obtained using the Delaunay triangulation (Jurczyk et al., 2000). Construction of the network starts by creating one or two triangles. The network is then expanded by adding more edge nodes and then a local reconfiguration of the network is performed.

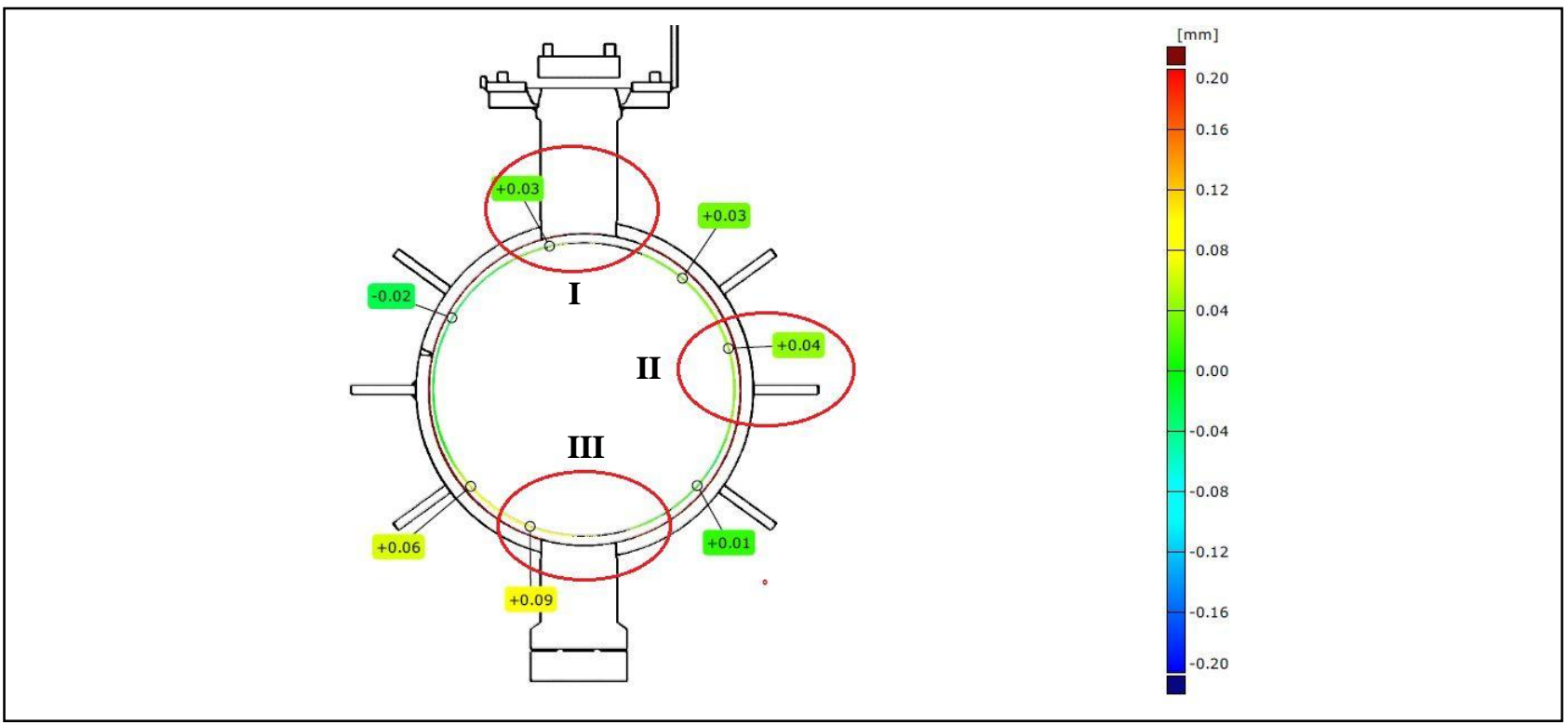

Fig. 5. Cross-section of the damper seat

After the point cloud and the 3D model are mutually superimposed, missing parts of the surface are visible in locations that are hard to access. This is due to the limitations of the method, most importantly the requirement to manually perform additional scanner readouts in parts of the object that are hard to access, such as cavities, stacks, or gaps; however, repeated calculations performed for triangles that do not meet the preset conditions, the shortcomings were minimized. 
The main objective of the tests was to determine the leak-tightness of the interface between the seat and the damper. This is why the control and measurement process for the real model was started from an inspection of the shape and the internal dimensions of the damper seat. The first inspection cross-section was prepared on a plane that was parallel to the surface of the seat, as shown in Fig. 5.

The dimensional analysis indicates small deviations from the dimensions of the theoretical model; however, the values of those dimensions are within the tolerances. Due to the complex closing process and the eccentric mounting of the damper, lack of leak-tightness in measurement areas where identified for the leak-tightness analysis. Fig. 6 shows selected measurement areas I-III. The measurements performed in selected cross-sections demonstrated the presence of significant differences in dimensions. The values of the measurement errors were equal to $0.1 \mathrm{~mm}$ to as much as $0.4 \mathrm{~mm}$. Cavities were present on surfaces that are perpendicular to the measurement plane and excess material was presented on surfaces that were parallel to the measurement plane.

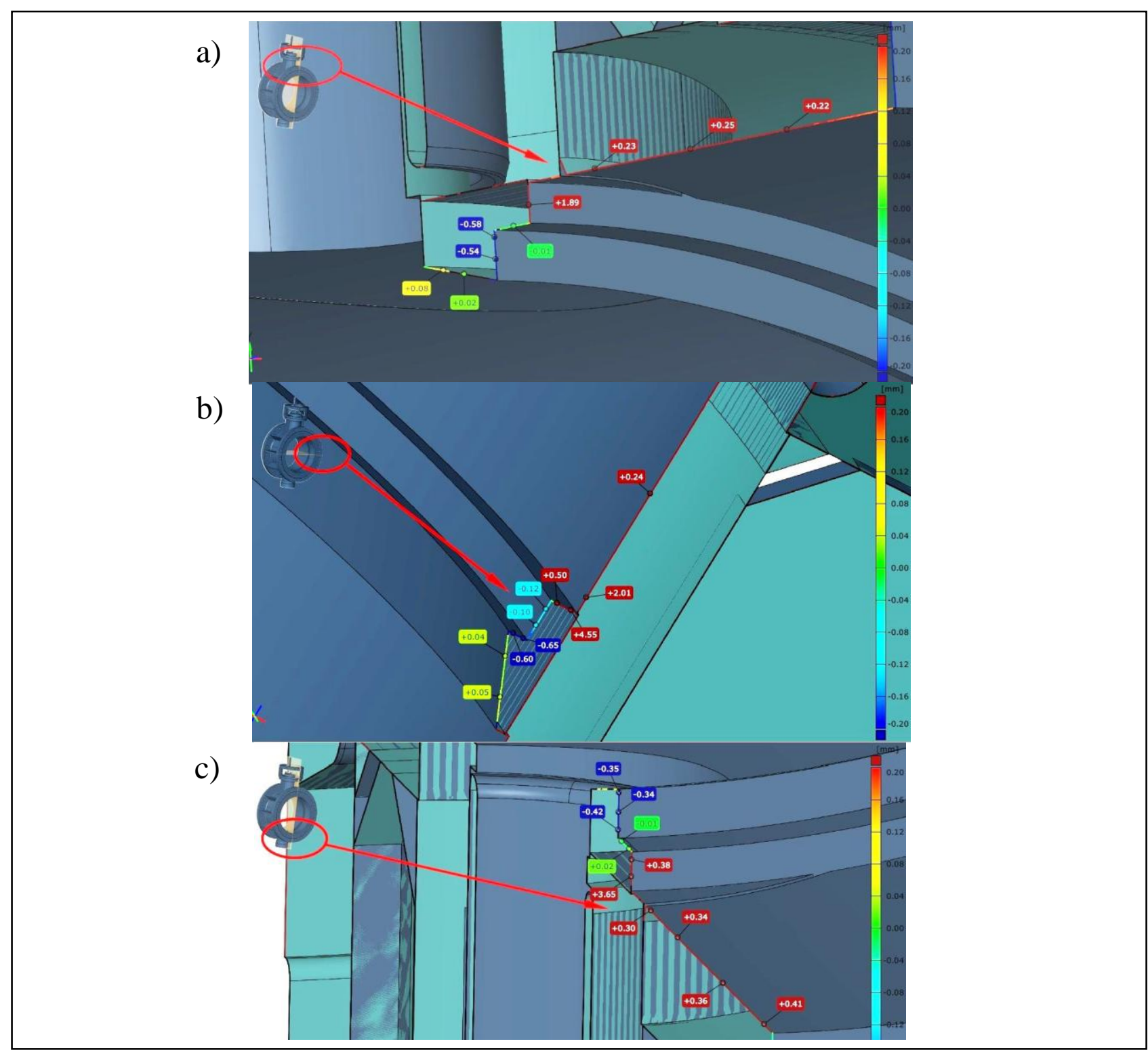

Fig. 6. Measurement areas - $a, b$ and c 
The last stage of the test involved an inspection of the position of the damper during the process of full closing and full opening (Fig. 7).

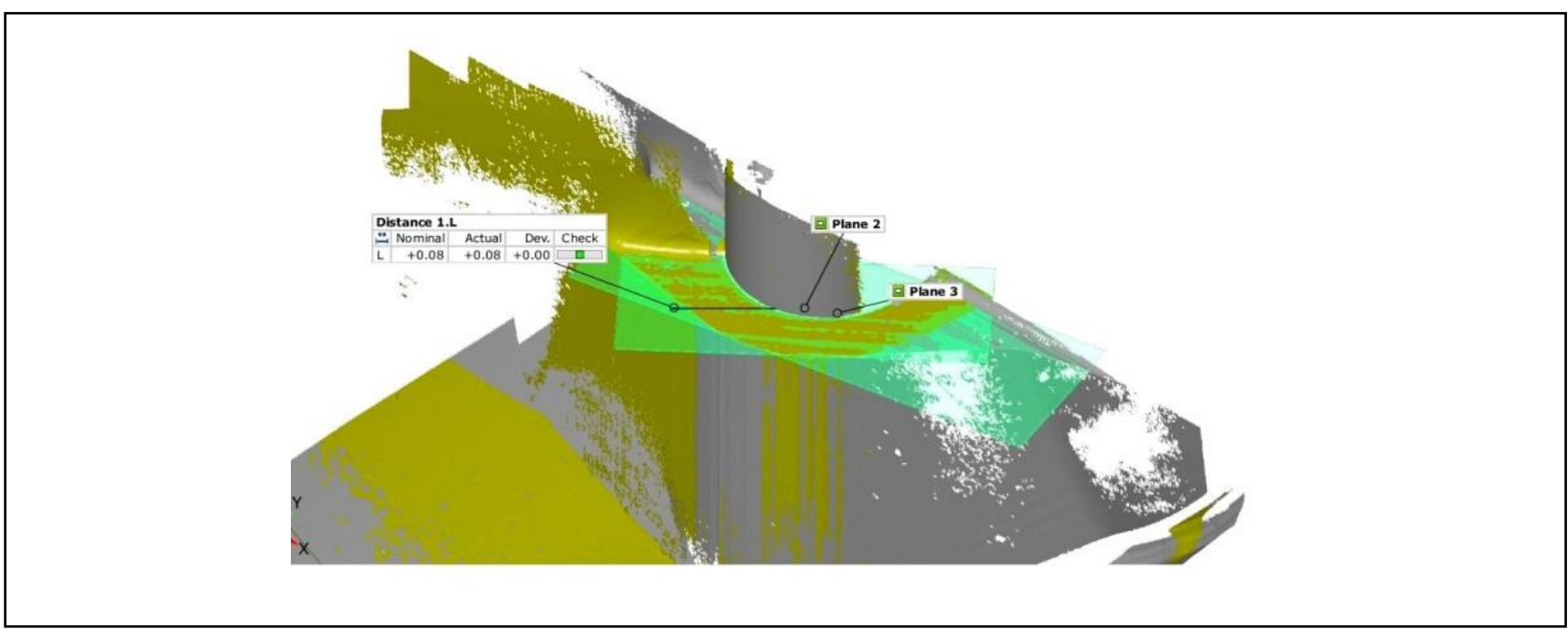

Fig. 7. Dimension between opened and closed damper

The measurement analysis demonstrated that the dimension between the closed damper and the opened damper is within the tolerances and the minimum distance is $0.1 \mathrm{~mm}$.

\section{Discussion}

The measurement process was performed in a manufacturing environment, which is a big advantage of the method used. The measurement field used enabled determination of the dimensions with the accuracy of up to $0.01 \mathrm{~mm}$. This range is sufficient for the purpose of evaluation of the tested object. The measurement points obtained in the form of a point cloud provide the full image of the tested geometric structure. However, processing of the point cloud may have a negative impact on the final measurement results (Zator \& Michalski, 2012). This is because point cloud processing software uses triangular networks to elaborate the geometry as well as average and median value filters. This is very important in the case of precise measurements; however, the impact of the tested object is not significant.

The tested damper is a prototype and its opening diameter is $500 \mathrm{~mm}$. Because the manufacturer assumes production of regulated dampers of larger diameters, in order to reduce the measurement process construction a model surface was recommended. The concept of construction of a modeler will be based on preparation of model surfaces that will be used as a basis for calibration of the measurement equipment. Model surfaces are surfaces whose geometry has a significant impact on the leak-tightness of the manufactured dampers. Preparation of such surfaces will enable performing precise measurements by using a coordinate measuring machine. The recommended concept of construction of a modeler will significantly reduce the measurement time and, most importantly, will increase the accuracy of the measurement. 


\section{Conclusion}

The measurement method presented in the paper, which uses the optical scanning technique, can be applied more broadly in manufacturing environments. The coordinate means machines that are being used currently must be utilized in special rooms and the measurement of large surfaces may be difficult. However, the advantage of 3D scanners is that they enable "touch-free" determination of real spatial objects. Coordinate measurement machines are very accurate. On the other hand, the contemporary structural light 3D scanners can achieve accuracy on the level of $0.01 \mathrm{~mm}$. Processing of a point cloud also has a negative impact on the measurements due to the averaging of the coordinates of points. Another disadvantage of $3 \mathrm{D}$ scanners is the fact that it is impossible to test many parameters of the geometric structure condition at the same time (Vacharanukul \& Mekid,2005, Shirashi,1989). However, the main objective of 3D scanning is to compare the condition of real surfaces to the condition of virtual surfaces. Such comparison can be considered to be active control that enables achieving high quality and repeatability of manufactured products, leads to a reduction in the number of defects, reduces the time needed to make a single component, and optimizes the operation time of the machining tools.

The results for the tested object presented herein demonstrate high measurement uncertainty; thus, a lower measurement uncertainty can be achieved by using a modeler. The use of a modeler will significantly improve the measurement process by enabling the performance of independent scans in specific locations. This will result in a reduced measurement uncertainty.

\section{References}

Glut, B.; Boryczko, K.; Jurczyk, T.; Alda, W. \& Kitowski, J. (2000), High quality 2D mesh generation based on Delaunay triangulation. In M. Bubak, J. Moscinski, and M. Noga, editors, Proc. SGI Users' Int. Conf. SGI2000, pp. 334-343, 2000, Krakow

Kesik, J. (2011), Project of the 3D scanning system utilizing stereovision and rotational platforms, Pomiary Automatyka Kontrola, Vol 57, No 12, 2011, ISSN 0032-4110, pp. 1483-1485

Rak, M. \& Wozniak, A. (2013), The influence of point cloud processing on the accuracy of determining a small element dimension, Pomiary Automatyka Kontrola, Vol 59, No 5, 2013, ISN S0032-4140, pp. 406-409

Shirashi, M. (1989), Scope of In-process Measurement, Monitoring and Control Techniques in Machining Processes - Part 2: In-process Techniques for Workpieces. Precision Engineering, Vol. 11, No. 1, 1989, pp. 27-37

Vacharanukul, K. \& Mekid, S. (2005), In-process Dimensional Inspection Sensors. Measurement, Vol. 38, No. 3, 2005, pp. 204-218

Zator, S. \& Michalski, P. (2012), Possibilities of using a laser scanner for precise measurements, Pomiary Automatyka Kontrola, Vol 58, No 11, 2012, ISN S00324140, pp. 1013-1015

*** http:// www.gom.com - Optical Measuring Techniques 